\title{
PRODUCT SOLUTIONS FOR SIMPLE GAMES. II
}

\section{T. PARTHASARATHY}

1. Introduction. In this paper we continue our study of the investigation of product solutions for compound simple games. By a compound simple game we mean one that is built up out of two or more component simple games. The concept of compound simple games is apparently due to Shapley. Shapley [2] and the author [1], [4], [5] have obtained product solutions for compound simple games by combining the solutions of the component games. During this process we impose that the subsolutions will have to satisfy a certain semimonotonic $\partial$-monotonic property. In this paper we obtain a new class of product solutions for the game $H \otimes K$ with $H=V_{n} \otimes B_{1}$, where $V_{n}$ denotes the homogeneous weighted majority game $[1,1,1, \cdots$, $1, n-2]_{h}$ consisting of $n$ players, $B_{1}$ denotes the 1 -person pure bargaining game and $K$ any arbitrary simple game.

\section{Definitions and notations.}

Simple Games. We shall denote a simple game by the symbol, $\Gamma(P, W)$, where $P$ is a finite set (players) and $W$ is a collection of subsets of $P$ (the winning coalitions). We demand that $P \in W$ and the empty set is not an element of $W$.

Let $\Gamma\left(P_{1}, W_{1}\right)$ and $\Gamma\left(P_{2}, W_{2}\right)$ be two simple games with $P_{1} \cap P_{2}=\varnothing$ and let $P=P_{1} \cup P_{2}$. Then the product $\Gamma\left(P_{1}, W_{1}\right) \otimes \Gamma\left(P_{2}, W_{2}\right)$ (for simplicity we will write $\left.P_{1} \otimes P_{2}\right)$ is defined as the game $\Gamma(P, W)$ where $W$ consists of all $S \subseteq P$ such that $S \cap P_{i} \in W_{i}$ for $i=1,2$. By an imputation we mean a real nonnegative vector $x$ such that $\sum_{i \in P x_{i}}=1$. $A_{P}$ will stand for the collection of all imputations. We recall that a solution of the game $\Gamma(P, W)$ is a set $X$ of imputations such that $X=A_{P}-\operatorname{dom} X$ where dom $X$ denotes the set of all $y \in A_{P}$ such that for some $x \in X$, the set $\left\{i \mid x_{i}>y_{i}\right\}$ is an element of $W$. The notations $\operatorname{dom}_{1}$ and $\operatorname{dom}_{2}$ will be used for domination with respect to special classes $W_{1}$ and $W_{2}$.

DEFinition. A parameterized family of sets of imputations $Y(\alpha): 0 \leqq \alpha \leqq 1$ will be called semimonotonic if for every $\alpha, \beta, x$ such that $0 \leqq \alpha \leqq \beta \leqq 1$ and $x \in Y(\beta)$ there exists $y \in Y(\alpha)$ with $\alpha y \leqq \beta x$.

Definition. A semimonotonic family is called $\partial$-monotonic $(0 \leqq \partial \leqq 1)$ if for every $\alpha, \beta, y$ such that $\partial \leqq \alpha \leqq \beta \leqq 1$ and $y \in Y(\alpha)$ there exists $x \in Y(\beta)$ with $\alpha y \leqq \beta x$.

Received by the editors November 30, 1966. 
We call a 0 -monotonic family fully monotonic. [In general, $\partial$ will stand for any positive number with $0<\partial<1$ unless otherwise stated.]

Let $P=P_{1} \cup P_{2}$ and let

$$
A_{P_{i}}=\left\{x: x \in A_{P}, \sum_{j \in P_{i}} x_{j}=1\right\} \quad \text { for } i=1,2 \text {. }
$$

Definition. Let $X$ be a solution to the product of simple games $P_{1} \otimes P_{2}$. Call $X$ a product solution if the following conditions are met.

(i) There exists a semimonotonic family $\left\{Y_{i}(\alpha): 0 \leqq \alpha \leqq 1\right\}$ such that $Y_{i}(\alpha)$ are solutions to $P_{i}$ for all $\alpha$ except $\alpha=1$ where $i=1,2$.

(ii) $X=\bigcup_{0 \leq \alpha \leq 1} X_{1}(\alpha) \times_{\alpha} X_{2}(1-\alpha)$ where $X_{i}(\alpha)=A_{P_{i}}-\operatorname{dom}_{i} Y_{i}(\alpha)$ and $X_{1}(\alpha) X_{\alpha} X_{2}(1-\alpha)=\left\{z: z=\alpha x_{1}+(1-\alpha) x_{2}\right.$ for some $x_{1} \in X_{1}(\alpha)$, $\left.x_{2} \in X_{2}(1-\alpha)\right\}$.

Definition. Let $X$ be any subset of $A_{P}$. Call $X$ an externally stable set if $X \cup \operatorname{dom} X=A_{P}$. Call $X$ an internally stable set if $X \cap \operatorname{dom} X$ $=\varnothing$. [Here of course we assume $\Gamma(P, W)$ to be a simple game.] Call $X$ a solution if $X$ is both externally stable and internally stable. $V_{n}$ will always stand for the homogeneous weighted majority game $[1, \cdots, 1, n-2]_{h}$ consisting of $n$ players. $H$ will stand for any game of the form $V_{n} \otimes B_{1}$ where $B_{1}$ denotes the 1 -person pure bargaining game. $K$ will denote an arbitrary simple game.

3. We now write down the solutions of $V_{n}$ which are completely known [3, pp. 472-495]. They are classified in three groups.

I. The finite set

$$
\begin{aligned}
& \left(\frac{1}{n-1}, \frac{1}{n-1}, \ldots, \frac{1}{n-1}, 0\right), \quad\left(\frac{1}{n-1}, 0,0,0,0, \frac{n-2}{n-1}\right), \\
& \left(0, \frac{1}{n-1}, \cdots, 0, \frac{n-2}{n-1}\right), \cdots, \quad\left(0,0,0,0, \cdots, \frac{1}{n-1}, \frac{n-2}{n-1}\right) .
\end{aligned}
$$

II. Let $C$ be any constant with $0 \leqq C<1-1 /(n-1)$

$$
\left\{\left(x_{1}, x_{2}, \cdots, x_{n-1}, C\right) \mid x_{i} \geqq 0, \sum x_{i}=1-C\right\} .
$$

III. Let $S_{*}$ be any nonempty proper subset of $\{1,2, \cdots, n-1\}$. Let $a_{1}, a_{2}, \cdots, a_{n-1}$ be nonnegative real numbers which satisfy the following properties:

(i) $\sum_{1}^{n-1} a_{i}=1$,

(ii) $a_{*}=\operatorname{Min}_{1 \leq i \leq n-1} a_{i}$, then $a_{i}=a_{*}$ for all $i \in S_{*}$ and $a_{i}>a_{*}$ for $i \in\{1,2, \cdots, n-1\}-S_{*}$.

As a consequence of (i) and (ii) we have $a_{*}<1 /(n-1)$. Let $p$ be 
the number of elements in $S_{*}$ and let $c=1-a_{*}, c^{*}=1-p a_{*}$. The following set consisting of (1) and (2) constitutes a solution to $V_{n}$.

(1) For $i \in S_{*}$,

where

$$
a^{i}=\left\{a_{1}^{i}, a_{2}^{i}, \cdots, a_{n}^{i}\right\}
$$

$$
\begin{aligned}
a_{j}^{i} & =a_{i}=a_{*} & & \text { for } j=i, \\
& =c & & \text { for } j=n, \\
& =0 & & \text { otherwise. }
\end{aligned}
$$

(2) $\boldsymbol{a}(y)=\left\{a_{1}(y), a_{2}(y), \cdots, a_{n}(y)\right\}$ where $0 \leqq y \leqq c^{*}$;

$$
\begin{aligned}
a_{i}(y) & =a_{i}=a_{*} & & \text { for } i \in S_{*}, \\
& =y & & \text { for } i=n, \\
& =a_{i}(y) & & \text { for } i \in\{1,2, \cdots, n-1\}-S_{*},
\end{aligned}
$$

where $a_{i}(y)$ for $i \in\{1,2, \cdots, n-1\}-S_{*}$ are functions whose domain of definition is $\left[0, c^{*}\right]$.They also have the following properties

$$
a_{i}(0)=a_{i}, \quad a_{i}\left(c^{*}\right)=0
$$

and

$$
\left|a_{i}\left(y_{2}\right)-a_{i}\left(y_{1}\right)\right| \leqq\left|y_{2}-y_{1}\right| \text {. }
$$

I, II and III exhaust all possible solutions to $V_{n}$.

REMARK 1. It is not hard to check that no semimonotonic family drawn from this list can include representatives from more than one of the three categories I-III, hence the only possible variation within such a family is in the value of $C$ if the family is from the second group or the variation will be in choosing $S_{*}$, the nonnegative real numbers $a_{1}, a_{2}, \cdots, a_{n-1}$ and the functions $a_{i}(y)$ for $i \in\{1,2, \cdots, n-1\}$ $-S_{*}$ if the family is from the third group.

REMARK 2. Setting $a_{*}=1 /(n-1)$ in III or II produces internally stable sets (not solutions) that are monotonically related to the solutions nearby. Using this fact we will give an example of a solution for compound simple games which is not fully monotonic in the last section. [Recall the fact that a set $X$ is internally stable if $X \cap \operatorname{dom} X=\phi$.

4. We will now state the theorems.

THEOREM 1. Let $\left\{X_{1}(\alpha): 0 \leqq \alpha \leqq 1\right\}$ be any $\partial$-monotonic family of product solutions to the game $H=V_{n} \otimes B_{1}$ except that $X_{1}(1)$ need not be externally stable. Then 


$$
X=\bigcup_{0 \leq \alpha \leq 1} Z_{1}(\alpha) \underset{\alpha}{\times} Z_{2}(1-\alpha)
$$

is a solution for $H \otimes K$ where $K$ is any arbitrary simple game and $Z_{1}(\alpha)=A_{n+1}-\operatorname{dom}_{1} X_{1}(\alpha)$ and $Z_{2}(1-\alpha) \equiv Z_{2}$ is any solution of $K$.

THEOREM 2. Let $Y_{1}(\alpha)$ be $\partial$-monotonic solutions to $V_{n}$. Then

$$
X=\bigcup_{0 \leq \alpha \leq 1} Z_{1}(\alpha) \underset{\alpha}{\times} Z_{2}(1-\alpha)
$$

is a solution for $V_{n} \otimes K$ where $Z_{1}(\alpha)=A_{n}-\operatorname{dom}_{1} Y_{1}(1)$ and $Z_{2}(\alpha) \equiv Z_{2}$ is any solution of $K$.

REMARK 3. External stability of Theorems 1 and 2 can be established as in the case of Theorem 5 of Shapley (see [2, pp. 282-283]) or as in [1] since the proof depends only on the semimonotonic property of $X_{i}(\alpha)$ and the external stability of $Z_{i}(\alpha)$.

Remark 4 . Theorem 2 does not say much. This is because every solution that satisfies the conditions of Theorem 2 in fact has the property of full monotonicity even in the 'not required' range. However, using Theorem 1, we can construct solutions which will be $\partial$-monotonic but not fully monotonic.

Proof of Theorem 1. We will now show that $X$ is internally stable. We will give the proof when $H=V_{4} \otimes B_{1}$. The same proof with some minor modifications applies when $H=V_{n} \otimes B_{1}$ for general $n$.

Case 1. Suppose for infinitely many $m$, with $\alpha^{(m)} \uparrow 1, X_{1}\left(\alpha^{(m)}\right)$ is of the form

$$
\begin{aligned}
X_{1}\left(\alpha^{m}\right)= & \left\{\beta\left(\frac{1}{3}, \frac{1}{3}, \frac{1}{3}, 0\right), 1-\beta\right\} \\
& \cup\left\{\beta\left(\frac{1}{3}, 0,0, \frac{2}{3}\right), 1-\beta\right\} \\
& \cup\left\{\beta\left(0, \frac{1}{3}, 0, \frac{2}{3}\right), 1-\beta\right\} \\
& \cup\left\{\beta\left(0,0, \frac{1}{3}, \frac{2}{3}\right), 1-\beta\right\} \quad \text { where } \beta \text { runs over from } 0 \text { to } 1 .
\end{aligned}
$$

This representation is possible since we are assuming that the $X_{1}(\alpha)$ 's are product solutions. In this case, since the family is $\partial$-monotonic and since all but a finite number of $\alpha^{(m)}$ 's are greater than $\partial$ it follows that $X_{1}(1)$ is also of the same form as $X_{1}\left(\alpha^{(m)}\right)$ 's. In other words $X_{1}(1)$ is a solution. Hence internal stability follows via Theorem 5 of Shapley [2].

Case 2. Let $\alpha^{(m)} \uparrow 1$ and

$$
\begin{aligned}
& X_{1}\left(\alpha^{(m)}\right)=\left\{\beta\left(x_{1}, x_{2}, x_{3}, C_{\beta}^{(m)}\right), 1-\beta \mid 0 \leqq \beta<1,\right. \\
&\left.x_{i} \geqq 0, \sum x_{i}=1-C_{\beta}^{(m)}\right\} \cup Y_{1}^{\alpha^{m}}(1),
\end{aligned}
$$


where $Y_{1}^{\alpha^{m}}(1)$ need not be an internally stable set for $V_{4}$ and $0 \leqq C_{\beta}^{m}$ $<2 / 3$. Consider the set $N_{\beta}$ where

$N_{\beta}=\left\{x \mid x=\beta\left(x_{1}, x_{2}, x_{3}, C_{\beta}^{0}\right), 1-\beta\right.$ and there exists a sequence

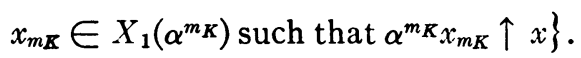

It is easy to check that $N_{\beta}$ is nonempty.

It is not hard to check that the closure of $X_{1}(1)-$ written as $\bar{X}_{1}(1)$ - contains the set $N_{\beta}$. This is a consequence of the assumption that the family $X_{1}(\alpha)$ is $\partial$-monotonic.

At this point we would like to make another observation, namely $\bar{X}_{1}(1)$ together with $\left\{X_{1}(\alpha): 0 \leqq \alpha<1\right\}$ is a semimonotonic family and hence $\bar{X}_{1}(1)$ is also internally stable.

If $C_{\beta}^{0}<2 / 3$ for every $\beta, \cup N_{\beta}=\bar{X}_{1}(1)$ and $\bar{X}_{1}(1)$ is a solution for $H$, internal stability follows by the theorem of Shapley.

Let $C_{\beta}=2 / 3$ for at least one $\beta$.To complete the proof of internal stability in his case it is sufficient to establish that there does not exist any vector $x \in X_{1}(\alpha)$ with $\alpha x$ dominating $y$ where $y \in Z_{1}(1)$ $-\bar{X}_{1}(1)$. Let if possible,

$$
\alpha x>y \text { via } \overline{145} \text { with } y \in Z_{1}(1)-\bar{X}_{1}(1) .
$$

$\left[\alpha x>y\right.$ via $\overline{145}$ means, $\alpha x_{i}>y_{i} \forall i=1,4,5$.]

Let $y=\left(\epsilon_{1}, \epsilon_{2}, \epsilon_{3}, \epsilon_{4}, \epsilon_{5}\right)$ and

$$
X(\alpha)=\left[\beta\left(x_{1}, x_{2}, x_{3}, C_{\beta}\right), 1-\beta\right] .
$$

[For the sake of simplicity we will not write the possible values of $\beta$ and $x_{1}, x_{2}, x_{3}$.]

Choose any $\beta^{\prime}$ with $\alpha(1-\beta)>1-\beta^{\prime}>\epsilon_{5}$ where

$$
\alpha\left[\beta\left(x_{1}^{\prime}, x_{2}^{\prime}, x_{3}^{\prime}, C_{\beta}\right), 1-\beta\right]>y \text { via } \overline{1} \overline{4} \overline{5} \text {. }
$$

Let $N_{\beta^{\prime}}=\left[\beta^{\prime}\left(x_{1}, x_{2}, x_{3}, C_{\beta^{\prime}}^{0}\right), 1-\beta^{\prime}\right]$. If $\beta^{\prime} C_{\beta^{\prime}}^{0} \leqq \epsilon_{4}$, then there exist $w \in N_{\beta}$, such that $\alpha x>w$ via $\overline{145}$. This will mean $\alpha x>w \geqq \alpha z$ via $\overline{145}$, contradicting the internal stability of $X_{1}(\alpha)$.

Hence we will assume $\beta^{\prime} C_{\beta \prime}^{0}>\epsilon_{4}$. This means $\beta^{\prime}\left(1-C_{\beta^{\prime}}^{0}\right)$ is less than or equal to $\epsilon_{1}, \epsilon_{2}$ and $\epsilon_{3}$, otherwise there will be an element in $N_{\beta^{\prime}}$ dominating $y$, thereby contradicting the assumption regarding $y$. Now a suitable $x^{1} \in X(\alpha)$ can be obtained with

$$
\alpha x^{\prime}>\left\{\beta^{\prime}\left(1-C_{\beta^{\prime}}^{0}, 0,0, C_{\beta^{\prime}}^{0}\right), 1-\beta^{\prime}\right\} \text { via } \overline{12} \overline{35}
$$

which will once again contradict the internal stability of $X_{1}(\alpha)$. Similar contradictions can be reached if $\alpha x>y$ via $\overline{245}$ or $\overline{345}$ or $\overline{1235}$.

Thus the proof of internal stability is complete in the case. 
Case 3. Let $\alpha^{(m)} \uparrow 1$ and

$$
X_{1}\left(\alpha^{(m)}\right)=\left[\beta\left(\stackrel{(m)}{a}, \stackrel{(m)}{a} \underset{\beta}{a}, \stackrel{(m)}{a} a_{\beta}(y), y\right), 1-\beta\right] \cup Y_{1}^{\alpha^{(m)}}(1)
$$

where $0 \leqq \beta<1, Y_{1}^{\alpha^{m}}(1)$ need not be an internally stable set for $V_{4}$ and $y$ runs from 0 to $1-2 a_{\beta}^{(m)}$ for every fixed $\beta$. Also note that $0 \leqq a_{\beta}^{(m)}<1 / 3$.

Let (w.l.g.) $a_{\beta}^{m} \rightarrow a_{\beta}^{0}$. Consider now the following set $N_{\beta}$

$$
N_{\beta}=\left\{x \mid x=\left\{\beta\left(a_{\beta}^{0}, a_{\beta}^{0}, a_{\beta}^{0}(y), y\right), 1-\beta\right\}\right.
$$

and there exists $x^{m_{k}} \in X_{1}^{\alpha_{m_{k}}}$ such that $\alpha^{\left.m_{k} x^{m_{k}} \uparrow x\right\}}$.

For every $\beta,\left\{a_{\beta}^{m}(y)\right\}$ is a collection of equicontinuous and uniformly bounded functions defined over the compact set

$$
A=\bigcap_{m=1}^{\infty} A_{\beta}^{m} \text { where } A_{\beta}^{m}=\left[0,1-2 a_{\beta}^{m}\right] .
$$

It is trivial to check that this intersection is precisely the interval $\left[0,1-2 a_{\beta}^{0}\right]$. So we can assert without loss of generality $a^{m}(y) \rightarrow a_{\beta}^{0}(y)$ uniformly over $A$. If for every $\beta, a_{\beta}^{0}<1 / 3$, we are through. So we will assume $Z_{1}(1)-\bar{X}_{1}(1) \neq \varnothing$. We will now prove that there exists no vector $x \in X(\alpha)$ with $\alpha x$ dominating $y$ where $y \in Z_{1}(1)-\bar{X}_{1}(1)$. Let if possible

i.e.

$$
\alpha x>y=\left(\epsilon_{1}, \epsilon_{2}, \epsilon_{3}, \epsilon_{4}, \epsilon_{b}\right) \text { via say } \overline{1235}
$$

$$
\alpha\left[\beta\left(x_{1}, x_{2}, x_{3}, x_{4}\right), 1-\beta\right]>y .
$$

Let $X_{1}(\alpha)$ be of the form

$$
\left\{\beta\left(a_{\beta}, a_{\beta}, a_{\beta}(t), t\right), 1-\beta\right\} .
$$

Choose and fix one $\beta^{\prime}$ such that $\alpha(1-\beta)>1-\beta^{\prime}>\epsilon_{5}$

$$
N_{\beta^{\prime}}=\left\{\beta^{\prime}\left(a_{\beta^{\prime}}^{0}, a_{\beta^{\prime}}^{0}, a_{\beta^{\prime}}^{0}(t), t\right), 1-\beta^{\prime}\right\} .
$$

If $\beta^{\prime} a_{\beta^{\prime}}^{0} \leqq \epsilon_{1}$ and $\epsilon_{2}$ then internal stability of $X_{1}(\alpha)$ will be contradicted. Hence, we will assume $\beta^{\prime} a_{\beta^{\prime}}^{0}>\epsilon_{1}$. This will mean $\beta^{\prime}\left(1-2 a_{\beta^{\prime}}^{0}\right) \leqq \epsilon_{4}$ otherwise there will be an element $w \in N_{\beta^{\prime}}$ such that $w$ will dominate $y$ thereby contradicting the assumption regarding $y$. If $\beta^{\prime} a_{\beta^{\prime}}^{0}>\epsilon_{2}$ and $\beta^{\prime}\left(1-2 a_{\beta^{\prime}}^{0}\right)>\epsilon_{3}$, then once again there will be a contradiction regarding the assumption that $y \in Z_{1}(1)-\bar{X}_{1}(1)$.

If $\beta^{\prime} a_{\beta}^{0} \leqq \epsilon_{2}$ then we can find $u, u^{\prime} \in X_{1}(\alpha), w \in N_{\beta^{\prime}}$ such that $\alpha u>w$ $\geqq \alpha u^{\prime}$ via 245 i.e. $u>u^{\prime}$ via 245 and this leads to a contradiction.

If $\beta^{\prime}\left(1-2 a_{\beta^{\prime}}^{0}\right) \leqq \epsilon_{3}$, we can find $u, u^{\prime} \in X(\alpha)$ with $u>u^{\prime}$ via $\overline{34} \overline{5}$ and hence a contradiction.

If $X_{1}(\alpha)$ is of the form 


$$
\left\{\beta\left(a_{\beta}, a_{\beta}^{1}(t), a_{\beta}^{2}(t), t\right), 1-\beta\right\},
$$

then also one can show the impossibility of $\alpha x$ dominating $y$ with $y \in Z_{1}(1)-\bar{X}_{1}(1)$. Similar contradictions can be reached if $N_{\beta^{\prime}}$ is of the form

$$
N_{\beta^{\prime}}=\left\{\beta^{\prime}\left(a_{\beta^{\prime}}^{0}, a_{\beta^{\prime}}^{1}(t), a_{\beta^{\prime}}^{2}(t), t\right), 1-\beta^{\prime}\right\} .
$$

Thus the proof of internal stability of $X$ is complete.

REMARK 5. During the course of the proof we have omitted certain minor details. For example if $X_{1}\left(\alpha^{\prime}\right)=\left\{\beta\left(x_{1}, x_{2}, x_{3}, c_{\beta}\right), 1-\beta\right\}$ then for all $\alpha$ with $\left(2 \alpha^{\prime} / 3\right)<\alpha \leqq \alpha^{\prime}, X(\alpha)$ will also be of the same form as $X\left(\alpha^{\prime}\right)$. This is a consequence of the fact that the $X(\alpha)$ 's form a semimonotonic family and are product solutions.

REMARK 6. Theorem 1 includes Theorem 3.2 in [1] where we have obtained product solutions for the game $H \otimes K, H=V_{3} \otimes B_{1}$ and $K$ is any simple game.

5. The following example shows that solutions to product simple games can be found which need not have the property of full monotonicity. Consider the game $H=V_{4} \otimes B_{1}$ and define for $0 \leqq \alpha \leqq 3 / 4$,

$$
X(\alpha)=\bigcup_{0 \leq \beta \leq 1} Y(\beta)
$$

where

$$
\begin{aligned}
& Y(\beta)=\left\{\left(\beta x_{1}, \beta x_{2}, \beta x_{3}, 2 \beta^{3 / 2} / 3,1-\beta\right) \mid x_{i} \geqq 0, \sum x_{i}=1-2 \beta^{1 / 2} / 3\right\} \\
& \quad \text { for } 0 \leqq \beta<1, \\
& Y(1)=\left\{\left(x_{1}, x_{2}, x_{3}, \frac{2}{3}, 0\right) \mid x_{i} \geqq 0, \sum x_{i}=\frac{1}{3}\right\} \cup\left\{\frac{1}{3}, \frac{1}{3}, \frac{1}{3}, 0,0\right\} .
\end{aligned}
$$

For $3 / 4<\alpha \leqq \alpha_{0}$, where $\alpha_{0}$ is so chosen that

$$
\alpha_{0}\left(1-\frac{7}{6} \frac{1}{1+\alpha_{0}}\right)=\frac{1}{3},
$$

define

$$
\begin{gathered}
X(\alpha)=\underset{0 \leq \beta \leq 1}{\bigcup} Y(\beta), \\
Y(\beta)=\left\{\left(\beta x_{1}, \beta x_{2}, \beta x_{3}, \frac{7}{6} \frac{\beta^{3 / 2}}{1+\alpha}, 1-\beta\right) \mid x_{i} \geqq 0,\right. \\
\left.\sum x_{i}=1-\frac{7}{6} \frac{\beta^{1 / 2}}{1+\alpha}\right\} .
\end{gathered}
$$

For $\alpha_{0}<\alpha \leqq 1$ define 


$$
\begin{array}{r}
X(\alpha)=\bigcup_{0 \leq \beta \leq 1}\left\{\left(\beta x_{1}, \beta x_{2}, \beta x_{3},\left(1-\frac{1}{3 \alpha}\right) \beta^{3 / 2}, 1-\beta\right) \mid x_{i} \geqq 0\right. \\
\\
\left.\sum x_{i}=1-\left(1-\frac{1}{3 \alpha}\right) \beta^{1 / 2}\right\} .
\end{array}
$$

Now it is not hard to check that the family $X(\alpha)$ is semimonotonic and that each $X(\alpha)$ is a product solution to the game $H$ except $X(1)$. $X(1)$ is not externally stable because,

$$
\left(\frac{1}{3}, \frac{1}{3}, \frac{1}{3}, 0,0\right) \notin X(1) \cup \operatorname{dom} X(1) .
$$

If $X^{1}(1)=X(1) \cup\left(\frac{1}{3}, \frac{1}{3}, \frac{1}{3}, 0,0\right)$, then $X^{1}(1)$ is a solution to $H$. But $X^{1}(1)$ together with $\{X(\alpha): 0 \leqq \alpha<1\}$ is not semimonotonic, for, corresponding to $\left(\frac{1}{3}, \frac{1}{3}, \frac{1}{3}, 0,0\right) \in X^{1}(1)$ there exists no element $x \in X(\alpha)$ for any $\alpha>3 / 4$ with $\alpha x \leqq\left(\frac{1}{3}, \frac{1}{3}, \frac{1}{3}, 0,0\right)$.

Further the family $X(\alpha): 0 \leqq \alpha \leqq 1$ is not fully monotonic because corresponding to the element $\left(\frac{1}{3}, \frac{1}{3}, \frac{1}{3}, 0,0\right) \in X(3 / 4)$ there exists no element $y \in X(1)$ with the property that $y \geqq(3 / 4)\left(\frac{1}{3}, \frac{1}{3}, \frac{1}{3}, 0,0\right)$. It is not hard to check that conditions of Theorem 1 are satisfied for this family $X(\alpha)$. Hence this family can be used to produce product solutions for the game $H \otimes K$. This is the example promised at the end of $\S 3$.

Acknowledgement. The author is greatly indebted to the referee for several useful suggestions.

\section{REFERENCES}

1. T. Parthasarathy, Minimax theorems and product solutions, Ph.D. Thesis, Indian Statistical Institute, Calcutta, 1966.

2. L. S. Shapley, Solutions of compound simple games, Annals of Math. Studies No. 52, Princeton Univ. Press, Princeton, N. J., 1964, pp. 267-305.

3. J. von Neumann and Oskar Morgenstern, Theory of games and economic behavior, Princeton Univ. Press, Princeton, N. J., 1944; Wiley, New York, 1964, pp. 472-495.

4. T. Parthasarathy, A note on compound simple games, Proc. Amer. Math. Soc., 17 (1966), 1334.

5. - Product solutions for simple games. I, Technical report, Mimeographed notes, Indian Statistical Institute, Calcutta, 1966.

Indian Statistical Institute, Calcutta and

University of California, Berkeley 